\title{
Interplay of electromagnetic noise and Kondo effect in quantum dots
}

\author{
Serge Florens, ${ }^{1,2}$ Pascal Simon, ${ }^{3,4}$ Sabine Andergassen, ${ }^{1}$ and Denis Feinberg ${ }^{1}$ \\ ${ }^{1}$ Institut Néel, Centre National de la Recherche Scientifique and Université Joseph Fourier, BP 166, 38042 Grenoble, France \\ ${ }^{2}$ Institut für Theorie der Kondensierten Materie, \\ Universität Karlsruhe, 76128 Karlsruhe, Germany \\ ${ }^{3}$ Laboratoire de Physique et Modélisation des Milieux Condensés, \\ Centre National de la Recherche Scientifique and Université Joseph Fourier, 38042 Grenoble, France \\ ${ }^{4}$ Department of Physics and Astronomy, University of Basel, \\ Klingelbergstrasse 82, CH-4056, Basel, Switzerland
}

\begin{abstract}
We investigate the influence of an electromagnetic environment, characterized by a finite impedance $Z(\omega)$, on the Kondo effect in quantum dots. The circuit voltage fluctuations couple to charge fluctuations in the dot and influence the spin exchange processes transferring charge between the electrodes. We discuss how the low-energy properties of a Kondo quantum dot subject to dynamical Coulomb blockade resemble those of Kondo impurities in Luttinger liquids. Using previous knowledge based on the bosonization of quantum impurity models, we show that low-voltage conductance anomalies appear at zero temperature. The conductance can vanish at low temperatures even in the presence of a screened impurity spin. Moreover, the quantitative determination of the corresponding Kondo temperature depends on the full frequency-dependent impedance of the circuit. This is demonstrated by a weak-coupling calculation in the Kondo interaction, taking into account the full distribution $P(E)$ of excited environmental modes.
\end{abstract}

\section{INTRODUCTION}

Recent progress in controlling the electronic properties of semiconductor-based nanostructures provides new ways of probing the physics of strong correlations, and envisions a rich interplay with truly mesoscopic effects. One of the most prominent examples is certainly the discovery of the Kondo effect in quantum dots $\stackrel{1,2}{=}$ While in metals containing a small amount of magnetic impurities the increased magnetic scattering of the electrons at low temperature results in an increased resistance, the mesoscopic realization displays instead a zero-bias peak in the conductance $\underline{3}^{3}$ This setup opens also a way for the study of various mesoscopic phenomena in the presence of strong correlations, such as nonequilibrium transport regimes $4.5,6$ and finite-size effects in the electrodes.., 8

One should emphasize that the unitary conductance, a feature in the charge sector, is currently taken as the fingerprint of the Kondo effect, a property of the impurity spin being screened by the conduction electron spins. Yet, it is not obvious that unitary conductance and spin screening should always coincide. In fact, one should bear in mind that conductance through a single or a double junction is sensitive to the circuit environment, made for instance of a resistance $R$. The parameter $r=R / R_{K}$ defines the normalized resistance with $R_{K}=$ $h / e^{2}$. For such an ohmic bath, in a strongly resistive single junction of resistance $R_{t}>>R_{K}$ and capacitance $C$, with charging energy $E_{c}=e^{2} / 2 C$, a low-bias anomaly $d I / d V \sim\left(V / E_{c}\right)^{2 r}$ appears. This phenomenon is called dynamical Coulomb blockade (DCB), (see Refs. 9, 10] for a review). It has been extensively studied in normal single tunnel junctions with resistive leads both experimentally $11,12,13$ and theoretically, $14,15,16,17,18,19,20,21,22$ as well as for general scatterers ${ }^{23,24}$, in normal double tun-

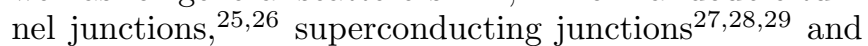

single barriers in semiconductors $\frac{30}{3}$ On the other hand, for transparent junctions with $R_{t}<R_{K}$, the same phenomenon occurs, but only below an exponentially small DCB energy scale $\tilde{E}_{C} \sim E_{c} e^{-R_{K} / 2 R_{t}}$. Only when at least one channel becomes fully transparent, dynamical Coulomb blockade disappears and a linear $I(V)$ is recovered $19,23,24,31,32$. Coming back to the Kondo problem, one readily sees the difficulty encountered in trying to guess the low-temperature behavior. On one hand, the zero-temperature regime being fully transparent, one might think that the Kondo effect - and the unitary conductance - are not affected by an ohmic bath. Yet, at any finite temperature, the transmission being less than one, DCB should reappear. In other words, it is not clear whether the Kondo temperature is lower or higher than a DCB energy scale. This problem obviously cannot be solved without reconsidering how the couplings leading to the Kondo effect (and the unitary-conductance peak) renormalize at low energy in presence of environmental fluctuations. This is the issue we address in the present paper.

Previous investigations of the Kondo effect with dynamical Coulomb blockade focused on the regime where the quantum dot is near the charge degeneracy point, with a noisy back gate directly coupled to the quantum dot $33,34,35,36$ In this case, a Kondo model for the charge degree of freedom can be derived, with a direct coupling of the dissipation to this charge variable. On general grounds this leads to a competition between the Kondo screening of the charge doublet by the electrons and the localization effect due to the ohmic environment.

Here, we concentrate rather on the usual spin Kondo effect, with environmental electromagnetic fluctuations in the electrodes. In this case, dissipation induces a markedly different effect on the Kondo physics. Indeed, only the inter-lead spin exchange processes involve charge 
fluctuations across the device and hence dominantly couple to the environment. One then assists to a competition between several Kondo-type strong-coupling fixed points in absence of localization effects. Different conduction regimes may then be approached, depending on the dissipation strength and microscopic couplings.

At weak dissipation, one expects to preserve the usual Kondo effect, but dephasing of the couplings involving charge transfer affects the determination of the Kondo temperature $T_{K}$ below which the spin is eventually screened on a quantitative level. For this purpose we examine how the perturbative regime of the Kondo model is modified by a general dissipative environment in the formalism of the $P(E)$ theory. Focusing on a circuit with a finite zero-frequency impedance $Z(\omega=0)=R$, but keeping a complete description of the environment characterized by the above low-energy ohmic behavior and a higher energy tail related to the circuit capacitances, we explicitely calculate how those parameters influence the temperature scale $T_{K}$ at which the Kondo resonance forms. At temperatures lower than $T_{K}$, we can apply previous knowledge $e^{37,38,39,40,41}$ on the Kondo effect between Luttinger liquids (to which our setup is equivalent in some low-energy limit only) to capture the currentvoltage relation. This extends the previous mappings for single or double junctions ${ }^{21,31}$. For the weak-dissipation regime, we thus find that nonohmic behavior, thus DCB, prevails in presence of particle-hole asymmetry. Particlehole symmetry can be restored for some specific values of the dot gate voltage allowing for a unitary conductance, albeit with anomalous corrections.

At large dissipation, which may be more challenging to realize experimentally, the electron transfer processes are even more strongly suppressed. As soon as the environmental impedance $R$ reaches half the quantum value $h / e^{2}$, a generic nonohmic transport regime develops (valid independently of particle-hole symmetry, but with unbalanced left/right couplings), where the Kondo effect occurs through the strongest coupled electrode only. With balanced couplings, a two-channel Kondo regime may be feasible, and represents a generalization of a recently proposed $\underline{42,43}$ and experimentally studied setup $\underline{44}$ with strong Coulomb blockaded leads, formally realizing the limit $r \gg 1$. We note that our proposal does not suffer from a low-energy cutoff set by the level spacing in the leads. Notice that weak dissipation $\left(R \ll R_{K}\right)$ is not sufficient to reach such non-Fermi liquid fixed point.

The paper is organized as follows. In Sec. II we give a characterization of the ohmic environment, and introduce the various physically relevant scales for the problem. In Sec. III we propose a phenomenological model describing how the Kondo effect may be affected by those environmental fluctuations. This model is analyzed both at weak coupling, taking into account the full spectral function $P(E)$ of the environment, and at strong coupling (zero temperature), using a low-energy mapping onto a Luttinger chain through bosonization, explicitly derived in Appendix A. We conclude the paper with an outlook on possible future theoretical developments and experimental signatures of dynamical Coulomb blockade on the Kondo physics.

\section{ENVIRONMENTAL COULOMB BLOCKADE}

\section{A. Circuit theory}

In this part we briefly summarize the basics of circuit theory $\underline{9.10}$ This enables us to introduce also some notations that will be used along the paper. The device we are interested in consists of a nano-object, which can be an artificial atom, a molecule or another interacting system, connected to reservoirs by two tunnel junctions. Let us call S this interacting system. Each time an electron tunnels in or out the system $\mathrm{S}$, this electron can excite modes in the electromagnetic environment, describing the circuit external to the tunnel junction. As the environment comprises $\mathrm{S}$ itself, the crucial part is to properly describe the tunnel junctions. A semi-phenomenological approach able to capture these effects has been developed by Nazarov $\underline{10,14}$ and Devoret et al..$\underline{17}$ The environment is modeled by its own impedance $Z(\omega)$ (now arbitrary), leading to an impedance $Z_{t}(\omega)$ seen by the junction. We denote by $Q$ the charge displacement at the surface of the capacitance caused by a tunneling event. Note that $Q$ is a collective variable. We also define $\varphi$ the phase conjugate to $Q$ satisfying $[\varphi, Q]=i e$. Let us now derive the correlation functions of the phase variable $\varphi$ in the case of ohmic fluctuations.

\section{B. Ohmic phase fluctuation spectrum}

Working with imaginary frequencies, we can parametrize the bosonic Green function ${ }^{45}$ for the phase variable as

$$
\begin{aligned}
G_{\varphi}(i \omega) & \equiv\langle\varphi(i \omega) \varphi(-i \omega)\rangle \\
& =2 \pi\left[\frac{R_{K}}{Z(i \omega)}|\omega|+R_{K} C \omega^{2}\right]^{-1},
\end{aligned}
$$

where the first contribution in Eq. (2) defines the the frequency-dependent impedance $Z(\omega)$ of the circuit $\stackrel{10}{\longleftarrow}$ while the second is associated with the junction capacitance $C$ (see Sec. IIIA for details). We take $\hbar=1$ in the following. For an ohmic environment, the circuit resistance is given by $R=Z(\omega=0)$, which from Eq. (2) yields the natural cutoff frequency $\omega_{R}=1 /(R C)$. However, even for strong dissipation $\left(R \sim R_{K}\right) \omega_{R}$ is a very large frequency, at which $Z(\omega)$ cannot be reduced to its zero-frequency value. The circuit impedance typically decays as $Z(\omega) \sim \sqrt{R /|\omega| C_{l}}=R \sqrt{\omega_{l} / \omega}$ for $\omega$ larger than $\omega_{l} ; \omega_{l}=1 /\left(R C_{l}\right)$, and $C_{l}$ is a measure of the leads capacitance $\underline{9}$ To simplify further calculations, we will mode ${ }^{46}$ this tail of the circuit impedance $Z(\omega)$ by 
a pure capacitive decay $Z(\omega) \sim R \omega_{l} / \omega$. This allows to rewrite the phase correlations as

$$
G_{\varphi}(i \omega)=2 \pi \frac{R}{R_{K}}\left[|\omega|+\frac{\omega^{2}}{\omega_{c}}\right]^{-1}
$$

where $\omega_{c}=\omega_{l} \omega_{R} /\left(\omega_{l}+\omega_{R}\right)$ is an effective cutoff. We introduce also the related effective capacitance $C_{\text {eff }}$ controlling the high-frequency tail of the phase fluctuations $\omega_{c}=1 /\left(R C_{\text {eff }}\right)$. Using simple complex analysis, we can extract the real-frequency spectral function

$$
\mathcal{I} m G_{\varphi}(\omega)=2 \pi \frac{R}{R_{K}}\left[\omega+\frac{\omega^{3}}{\omega_{c}^{2}}\right]^{-1}
$$

which obeys

$$
G_{\varphi}(i \omega)=-\int \frac{d \epsilon}{\pi} \frac{\mathcal{I} m\left[G_{\varphi}(\epsilon)\right]}{i \omega-\epsilon} .
$$

Fourier transforming in imaginary time $\tau$ yields

$$
G_{\varphi}(\tau)=-\int \frac{d \epsilon}{\pi} \frac{1}{e^{-\beta \epsilon}-1} \mathcal{I} m\left[G_{\varphi}(\epsilon)\right] e^{-\epsilon \tau}
$$

where $\beta=1 /\left(k_{B} T\right)$ is the inverse temperature, and performing the analytic continuation $\tau \rightarrow i t$ to real time $t$, we get the usual formula 10

$$
\begin{aligned}
J_{\varphi}(t) & \equiv\langle(\varphi(t)-\varphi(0)) \varphi(0)\rangle \\
& =\int_{0}^{\infty} \frac{d \omega}{\omega} \frac{2 \mathcal{R} e\left[Z_{t}(\omega)\right]}{R_{K}}\left(\frac{\cos (\omega t)-1}{\tanh (\beta \omega / 2)}-i \sin (\omega t)\right),
\end{aligned}
$$

where $Z_{t}(\omega)=[i \omega C+1 / R]^{-1}$ is the impedance seen by the junction. Since charge transfer processes are associated to the operator $e^{i \varphi}$ (see Sec. III A) , it is useful to consider the real-time correlation function $\left\langle e^{i \varphi(t)-i \varphi(0)}\right\rangle=$ $e^{J_{\varphi}(t)}$, which by Fourier transformation

$$
P(E)=\frac{1}{2 \pi} \int_{-\infty}^{\infty} d t e^{J_{\varphi}(t)+i E t}
$$

describes the probability $P(E)$ that the electron exchanges an energy $E$ with the environment during the tunneling process.

At zero temperature and for long time

$$
J_{\varphi}(t) \sim-2 r \ln \left(i \omega_{c} t\right),
$$

where $r=R / R_{K}$ is the dimensionless resistance of the circuit, which leads to the low-energy behavior ${ }^{10}$

$$
P(E) \sim A_{r} \frac{E^{2 r-1}}{\omega_{c}^{2 r}} \quad \text { for } \quad \mathrm{E} \ll \omega_{\mathrm{c}},
$$

with $A_{r}=e^{-2 r \gamma} / \Gamma(2 r)$. This power law is however cut off for energies larger than $\omega_{c}$; in this range the probability decays as

$$
P(E) \sim 2 r \frac{\omega_{c}^{2}}{E^{3}} \quad \text { for } \quad \mathrm{E} \gg \omega_{\mathrm{c}} .
$$

We finally mention some useful properties 10 of the $P(E)$ function: i) detailed balance $P(E)=0$ for $E<0$ at $T=0$; ii) normalization $\int_{0}^{+\infty} d E P(E)=1$; iii) integral equation

$$
E P(E)=2 r \int_{0}^{E} d \omega \frac{P(\omega)}{1+\left(\frac{\omega-E}{\omega_{c}}\right)^{2}} .
$$

In the next section we analyze how the Kondo effect occurring in mesoscopic quantum dots is modified by the presence of such an ohmic environment.

\section{THE NOISY KONDO EFFECT}

\section{A. Derivation of the model}

In this section, we consider a single quantum dot connected to two metallic leads in the Kondo regime, and investigate how the Kondo effect is affected by the presence of environmental Coulomb blockade. We assume that electromagnetic fluctuations are modeled by an impedance $Z(\omega) \sim R$ closing the circuit as described above. The equivalent circuit depicted in Fig. 1 is the one of a single-electron transistor.

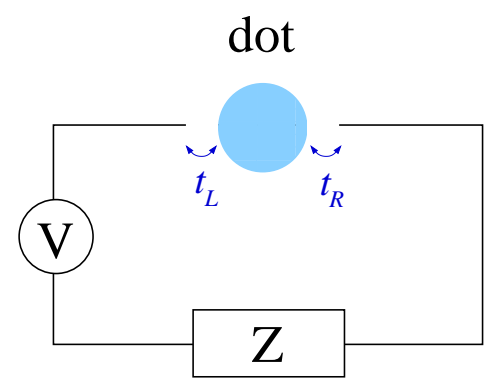

FIG. 1: (Color online) Schematic representation of a circuit with a dot coupled to two leads and an impedance $Z$.

A model Hamiltonian describing this situation can be written as $H=H_{\text {dot }}+H_{\text {leads }}+H_{\text {tun }}+H_{\text {bath }}$, where

$$
\begin{aligned}
H_{\mathrm{dot}} & =\sum_{\sigma} \epsilon_{d} d_{\sigma}^{\dagger} d_{\sigma}+U n_{d, \uparrow} n_{d, \downarrow}, \\
H_{\text {leads }} & =\sum_{k, \sigma, \alpha=L / R} \epsilon_{k} c_{k, \sigma, \alpha}^{\dagger} c_{k, \sigma, \alpha}, \\
H_{\text {tun }} & =\sum_{k, \sigma}\left(t_{L} e^{i \varphi_{L}} d_{\sigma}^{\dagger} c_{k, \sigma, L}+t_{R} e^{i \varphi_{R}} c_{k, \sigma, R}^{\dagger} d_{\sigma}\right)+\text { H.c.. }
\end{aligned}
$$

$H_{\text {bath }}$ describes the environmental degrees of freedom consisting of a collection of harmonic oscillators, alternatively expressed by a phase correlator such as Eq. (2). In these equations, $c_{k, \sigma, \alpha}^{\dagger}$ creates an electron with energy $\epsilon_{k}$ in lead $\alpha=L, R$ with spin $\sigma$, and $d_{\sigma}^{\dagger}$ creates an electron in the dot with spin $\sigma . H_{\text {dot }}$ is the usual Anderson 
Hamiltonian, where $\epsilon_{d}$ is the dot energy controlled by a gate voltage $V_{g}$, while $U$ describes the Coulomb interaction within the dot. The phases $\varphi_{\alpha}$ appearing in the tunneling Hamiltonian are related to the voltage fluctuations $\delta V_{\alpha}$ felt by an electron during a tunneling event through the $L / R$ junction as follows

$$
\varphi_{\alpha}(t)=e \int_{-\infty}^{t} \delta V_{\alpha}\left(t^{\prime}\right) d t^{\prime}
$$

The phases $\varphi_{\alpha}$ are conjugate to the charge $Q_{\alpha}$ on the junction capacitance $\alpha$ such that $\left[\varphi_{\alpha}, Q_{\alpha}\right]=i e$. As they originate from the same bath, the phases $\varphi_{\alpha}$ are clearly not independent. In order to relate the voltage fluctuations in the junction $\alpha$ to $Z(\omega)$, we use the standard effective-circuit description: 10 when an electron tunnels in the left junction, it feels an environment that can be modeled by the right junction in parallel with $Z(\omega)$. The left/right junctions are simply modeled by a tunneling resistance $R_{t}$ in parallel with a capacitance $C_{L} / C_{R}$. This description is consistent with the single-electron tunneling terms in Eq. (15), and may be derived more microscopically by coupling independently each of the tunneling operators to the bosonic charge modes in the circuit 25,47 . Neglecting the Kondo correlations for a moment, the setup of Fig. 1 is simply a double junction, which has been treated in great detail in Ref. [10] in the sequential regime. For a quantum mechanical description of the double junction in series, it turns out to be more convenient to work with the charges $q=Q_{L}-Q_{R}$ and $Q=\frac{C_{R} Q_{L}+C_{L} Q_{R}}{C_{L}+C_{R}}$ being uncoupled in the absence of tunneling; $q$ corresponds to the dot charge quantized in units of $e$ and $Q$ is simply the total charge carried by the capacitance $C=\frac{C_{L} C_{R}}{C_{L}+C_{R}}$ that couples directly to the impedance $Z$. One may then introduce the phases $\varphi$ and $\psi$ conjugate to $Q$ and $q$ respectively, related to the phases $\varphi_{\alpha}$ by $\varphi_{L}=\left(C / C_{L}\right) \varphi+\psi$ and $\varphi_{R}=-\left(C / C_{R}\right) \varphi+\psi$. In the equivalent circuit, we neglected the part corresponding to the gate voltage controlling the number of electrons on the dot. This approximation is justified, as the capacitance $C_{g}$ associated to the gate in general satisfies $C_{g} \ll C_{\alpha}$ and gate voltage fluctuations usually play a minor role (their contribution has been addressed in Ref. 48).

Since we are interested in the Kondo regime, where the real charge fluctuations in the dot are suppressed, it is convenient to perform a generalized Schrieffer-Wolff transformation, or equivalently integrate out the dot charge degrees of freedom. The complication due to the time-dependence of the tunneling term in Eq. (15) can be circumvented by a few additional assumptions. First we will assume that $|\delta V| \ll\left|\varepsilon_{d}\right|, U+\varepsilon_{d}$ which constitutes no serious limitation. We further suppose that the energy excitations of the environmental bosonic modes $\omega$ are small enough to satisfy $\omega \ll\left|\varepsilon_{d}\right|, U+\varepsilon_{d}$. Under these two conditions, the dot is modeled by a spin $\vec{S}$ described by a Kondo-like Hamiltonian $H_{K}$, involving phase-dependent couplings

$$
H_{K}=\sum_{\alpha, \beta} J_{\alpha, \beta} e^{i\left(\varphi_{\alpha}-\varphi_{\beta}\right)} \sum_{k, \sigma, k^{\prime} \sigma^{\prime}} c_{k, \sigma, \alpha}^{\dagger} \frac{\vec{\tau}_{\sigma, \sigma^{\prime}}}{2} c_{k^{\prime}, \sigma^{\prime}, \beta} \cdot \vec{S},
$$

where

$$
J_{\alpha, \beta} \approx 2 t_{\alpha} t_{\beta}\left(\frac{-1}{\varepsilon_{d}}+\frac{1}{U+\varepsilon_{d}}\right) .
$$

In Eq. (17) we should a priori include a dressed potential scattering term

$$
H_{V}=\sum_{\alpha, \beta} V_{\alpha, \beta} e^{i\left(\varphi_{\alpha}-\varphi_{\beta}\right)} \sum_{k, \sigma, k^{\prime} \sigma^{\prime}} c_{k, \sigma, \alpha}^{\dagger} c_{k^{\prime}, \sigma^{\prime}, \beta},
$$

where $V_{\alpha, \beta} \approx 2 t_{\alpha} t_{\beta}\left(\frac{-1}{\varepsilon_{d}}-\frac{1}{U+\varepsilon_{d}}\right)$. Nevertheless, the coupling constants $V_{\alpha \beta}$ turn out to be either irrelevant or marginal. As they are not modifying our weak-coupling analysis, we neglect these terms. However, these terms associated to particle-hole symmetry breaking are important near the strong-coupling fixed point as we will see.

The omission of the environment fluctuations with respect to $\epsilon_{d}$ or $U$ in Eq. (18) means that during a virtual charge fluctuation or cotunneling event $\underline{49}$ no energy is exchanged with the environment. This assumption is justified by the low value of the circuit cutoff frequency $\omega_{l}$. It contrasts for instance with previous treatments of so-called inelastic cotunneling 25 , or coupling with local phonon modes 50 . We expect that going beyond this approximation would lead to small corrections to the model defined above, and their treatment (see Ref. [50] for a different setup and simpler environment) is clearly beyond the scope of the present paper. Therefore, the elementary Kondo couplings result from quasi-"elastic" cotunneling (in the sense of Ref. [49]), where the initial and final states of the spin-flip transition may however involve different environmental energies. The inter-lead or "backscattering" Kondo couplings $J_{L R}$ and $J_{R L}$ now include the effects of the environment, embodied by the dynamical phase $\varphi_{L}(t)-\varphi_{R}(t)=\varphi(t)$. This dynamical phase clearly modifies the behavior of these couplings, which we will analyze in the next two subsections. On the contrary, within our quasi-elastic approximation, the intra-lead couplings $J_{L L}$ and $J_{R R}$ are not dressed by phase fluctuations.

\section{B. Weak Kondo coupling analysis}

\section{Poor-man's scaling}

The Hamiltonian (17) can be regarded as the usual Kondo model, where the inter-lead spin processes $J_{\alpha, \beta}$, with $\alpha \neq \beta$ transferring charge between the electrodes, are dressed by fluctuating phases described by the ohmic spectrum of Eq. (2). The case of strong Coulomb blockade, corresponding to $R=\infty$, has been previously studied in Ref. [43]. The calculation of observables to lowest 
order amounts to compute the diagrammatics at order $J^{2}$. Clearly the renormalization of the $J_{L R}$ vertex depends on the combination $J_{L R}\left(J_{L L}+J_{R R}\right)$, such that a single unpaired $e^{i \varphi}$ term occurs, and does not affect the usual renormalization equation

$$
J_{L R}^{\prime}=J_{L R}+J_{L R}\left(J_{L L}+J_{R R}\right) \int_{\Lambda-\delta \Lambda}^{\Lambda} d \epsilon \frac{\rho_{0}(\epsilon)}{-\epsilon}
$$

where $\rho_{0}(\epsilon)=1 / 2 W$ is the free electron density of states and $W$ is the electronic bandwidth. Following the poorman's scaling philosophy, we introduce here a small shift $\delta \Lambda$ in the running cutoff $\Lambda$ and computed the resulting change in the Kondo coupling. This flow starts at some high-energy initial cutoff $D \sim \min (\mathrm{U}, \mathrm{W})$, and reduces towards lower energies as long as it is perturbatively controlled. Introducing dimensionless couplings $j_{\alpha, \beta} \equiv \rho_{0} J_{\alpha, \beta}$ yields

$$
\frac{d j_{L R}}{d \log \Lambda}=-j_{L R}\left(j_{L L}+j_{R R}\right) .
$$

On the contrary, the $J_{L L}$ process is obtained from the combination $J_{L L}^{2}+J_{L R}^{2}$, so that the second term involves the combination of two exponential phase factors. This amounts to replace in the diagrammatics the free electronic Green function $G_{0}(\tau)=\left\langle c_{R}^{\dagger}(\tau) c_{R}(0)\right\rangle$ by a mixed one:

$$
\begin{aligned}
G_{m i x}(\tau) & =\left\langle c_{R}^{\dagger}(\tau) c_{R}(0) e^{i \varphi(\tau)} e^{-i \varphi(0)}\right\rangle \\
& \approx G_{0}(\tau) e^{J_{\varphi}(\tau)} .
\end{aligned}
$$

We denote $\rho_{\text {mix }}$ its associated density of states. Performing the analytic continuation of the above Green function at zero temperature, we find the simple formula

$$
\rho_{\text {mix }}(\omega)=\rho_{0} \int_{0}^{|\omega|} d E P(E) .
$$

The renormalization equation thus reads

$J_{L L}^{\prime}=J_{L L}+J_{L L}^{2} \int_{\Lambda-\delta \Lambda}^{\Lambda} d \epsilon \frac{\rho_{0}(\epsilon)}{-\epsilon}+J_{L R}^{2} \int_{\Lambda-\delta \Lambda}^{\Lambda} d \epsilon \frac{\rho_{\operatorname{mix}}(\epsilon)}{-\epsilon}$

leading to the flow equation

$$
\frac{d j_{L L}}{d \log \Lambda}=-j_{L L}^{2}-j_{L R}^{2} \int_{0}^{\Lambda} d E P(E) .
$$

In the following we analyze this result.

\section{General considerations}

The flow equations (21) and (26) are the main result of this Section. They describe the weak-coupling behavior of the Kondo model in a generic environment characterized by the $P(E)$ distribution, under the quasi-elastic approximation. We now specialize to the case of ohmic dissipation, for which $P(E)$ was discussed in Sec. [I]

Although the complete form of $P(E)$ is complicated, under the assumption that $\omega_{c} \ll U$ (consistent with the quasi-elastic approximation) we can roughly sketch the behavior of the flow in a simple two-step picture with respect to the energy scale $\omega_{c}$. At the beginning of the flow, $\Lambda \gg \omega_{c}$ and the energy integral in Eq. (26) is unity since $P(E)$ is a probability. One thus recovers the usual Kondo flow equations, i.e. dissipation is ineffective in this case. This can be understood by the disappearance of DCB at high energy, and will give rise to an initial strong renormalization of all Kondo couplings as if Coulomb blockade was absent.

This flow continues until $\Lambda$ reaches $\omega_{c}$, where the anomalous low-energy behavior of $P(E)$ given by Eq. (10) comes into play. At this stage, it is useful to introduce new dimensionless couplings $\lambda_{\alpha, \beta} \equiv \sqrt{A_{r} / 2 r}\left(\Lambda / \omega_{c}\right)^{r} j_{\alpha, \beta}$ for $\alpha \neq \beta$, and $\lambda_{\alpha, \alpha}=j_{\alpha, \alpha}$, which allow to rewrite the flow equations as

$$
\begin{aligned}
& \frac{d \lambda_{L R}}{d \log \Lambda}=r \lambda_{L R}-\lambda_{L R}\left(\lambda_{L L}+\lambda_{R R}\right) \\
& \frac{d \lambda_{L L}}{d \log \Lambda}=-\lambda_{L L}^{2}-\lambda_{L R}^{2} .
\end{aligned}
$$

Let us first give some general comments on these equations. First we notice that the dissipation affects the inter-lead couplings Eq. 27a and drives them irrelevant, at least as long as $\lambda_{L L}+\lambda_{R R}$ is not too large. This is consistent with the picture where charge transfer across the quantum dot is suppressed by DCB, and has the clear consequence of diminishing the Kondo temperature at increasing values of $r$ (see also the discussion in Sec. III B 3 and corresponding Fig. 2). Second, these flow equations are reminiscent of those obtained for the tunneling through a magnetic impurity in a Luttinger liquid,, 38 although we did not obtain them from bosonization. This identification will be put on rigorous grounds in Sec. IIIC by virtue of an exact mapping between the two problems. This also allows the computation of transport properties of the device at low temperature, that the weak-coupling calculation is unable to capture.

The above two-step argument thus shows that the slow onset of DCB at the scale $\omega_{c}$ reinforces the flow with respect to a pure Luttinger flow of the type (27), so that the decrease of the Kondo temperature with increasing dissipation will not be as pronounced as in the case of a pure Luttinger chain.

We also note that the cutting of $P(E)$ at $\omega_{c}$ is certainly not quantitative since $P(E)$ is a broad distribution, and we will solve the flow Eqs. (21) and (26) numerivally, with the full evaluation of $P(E)$ according to the integral Eq. (12), as performed below.

A final remark concerns the case of strong Coulomb blockade for $r \gg 1$. In this regime, the Coulomb charging energy $E_{c}=e^{2} / 2 C$ emerges as a natural cutoff ${ }^{56}$ with $P(E) \simeq \delta\left(E-E_{c}\right)$. For this case the two-step argument 
becomes exact,,$\underline{43}$ and leads to a strong renormalization of the Kondo temperature.

\section{Kondo temperature}

We start for simplicity by considering the solution of the pure Luttinger liquid case, described by Eqs. (27). This corresponds to the regime of large $\omega_{c}$, ignoring the mixing of boson excitation energies in the initial Kondo coupling. The results displayed in Fig. 2 however illustrate in the simplest manner the role of the parameter $r$. The first general effect is, as expected, a systematic decrease of the Kondo temperature $T_{K}$ with increasing $r$, marking a systematic onset of a strong-coupling regime. We note that $T_{K}$ remains nonzero even for strong dissipation, contrarily to the noisy "charge" Kondo effect, 33.34 in which voltage fluctuations act as a fluctuating magnetic field would act in the spin Kondo problem, that may prevent the formation of the Kondo resonance. Apart

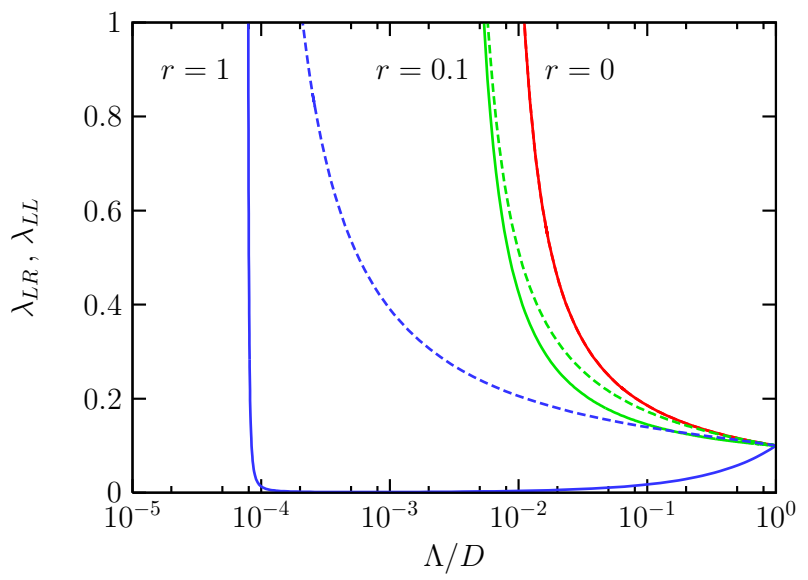

FIG. 2: (Color online) Flow of the Kondo couplings $\lambda_{L R}(\Lambda / D)$ (full line) and $\lambda_{L L}(\Lambda / D)$ (dashed line) according to Eqs. (27) (i.e. pure Luttinger liquids), for different values of $r$. The initial values of the Kondo couplings at scale $\Lambda=D$ are taken here as $\lambda_{L L}=\lambda_{L R}=0.1$ (with $L / R$ symmetry).

from this quantitative effect, we infer from Fig. 2 that dissipation can furthermore discriminate between several strong-coupling fixed points. Indeed when the impedance $R$ becomes comparable to the quantum value $R_{K}$, the inter-lead couplings $\lambda_{L R}$ are strongly driven to zero. This should clearly lead to qualitatively different transport properties compared to the case of weak dissipation. This also allows for a stabilization of a two-channel Kondo effect by DCB, for the case of balanced $\lambda_{L L}$ and $\lambda_{R R}$ couplings. These aspects are confirmed by the strongcoupling analysis performed in Sec. IIIC

The solution of the flow Eqs. (21) and (26) with ohmic dissipation, using the full $P(E)$ function determined by Eq. (12), is shown in Fig. 3. It illustrates the general fact that the full distribution function $P(E)$ of the environmental modes (or equivalently the complete frequency- dependent impedance of the junction in the circuit) affects the determination of the Kondo temperature in a significant quantitative manner. This is also seen by a

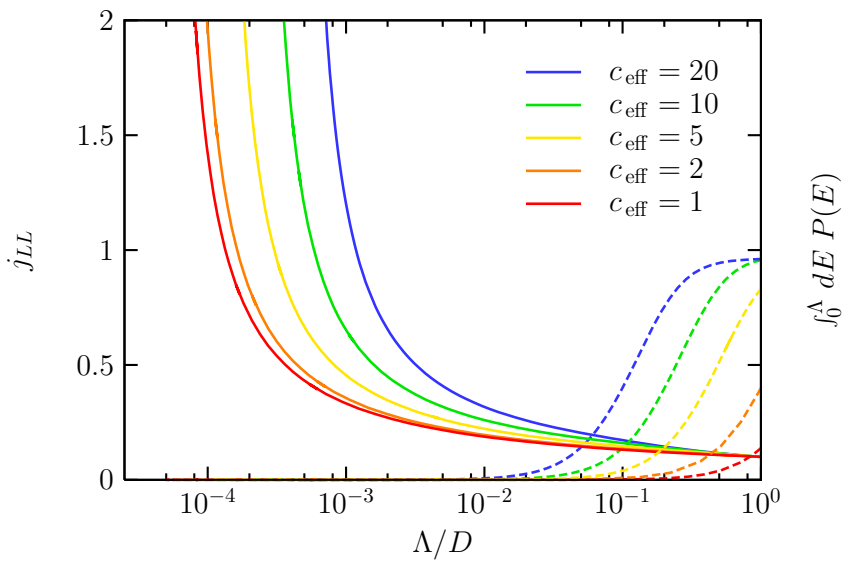

FIG. 3: (Color online) Flow of the Kondo coupling $j_{L L}(\Lambda / D)$ (full lines) according to Eqs. (21) and (26), and the corresponding integrated distribution $\int_{0}^{\Lambda} d E P(E)$ (dashed lines), for the dissipation strength $r=1$, initial couplings $j_{L L}=$ $j_{L R}=0.1$ and several values of the dimensionless effective capacitance $c_{\text {eff }}=D R_{K} C_{\text {eff }}=1,2,5,10,20$ (from bottom to top).

systematic study of the Kondo temperature (defined here by the criterion $\left.j_{L L}\left(\Lambda=T_{K}\right)=10\right)$ as a function of dissipation $r$ and for several values of the effective impedance

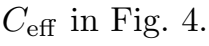

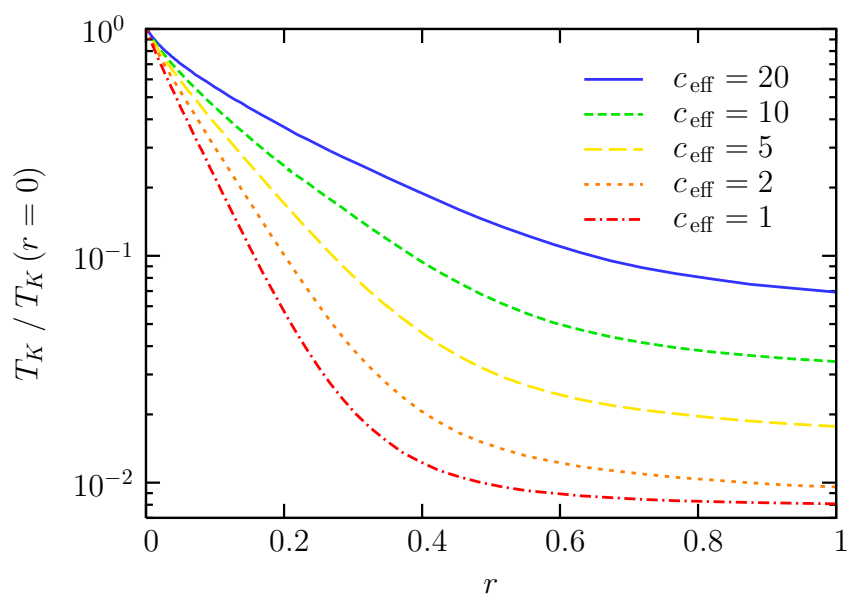

FIG. 4: (Color online) Kondo temperature $T_{K}$ as a function of dissipation $r$, for several values of the dimensionless effective capacitance $c_{\text {eff }}=1,2,5,10,20$ (from bottom to top). 


\section{Transport anomalies}

\section{Equivalence to the Kondo model between Luttinger liquid leads}

The previous weak-coupling analysis already informs about the type of ground state favored by the environment. More sophisticated tools are however necessary to make precise statements on the nature of the ground state, and to compute low-temperature and low-voltage properties. Here we will formulate the low-energy equivalence between the Hamiltonian (17) and the problem of a $S=1 / 2$ magnetic impurity weakly coupled to two Luttinger liquids, in the tunneling geometry. This equivalence has been already shown at the Hamiltonian level for a nonmagnetic impurity by Safi and Saleur ${ }^{21}$ (see also Ref. [34]), and can be easily extended to the Kondo model through bosonization of the lead electrons, $\frac{32}{2}$ as derived in App. A. The final result of this calculation allows to identify the effective interaction parameter of the Luttinger chain as

$$
K=\frac{1}{1+2 r} .
$$

The problem of a magnetic impurity in a Luttinger liquid has been analyzed using weak-coupling renormalizationgroup equations and a stability analysis of the strongcoupling limit by Fabrizio and Gogolin ${ }^{38}$, see also Ref. [39] for a conformal field theory analysis. It is thus not surprising that the low-energy limit (27) of the flow Eqs. (21) and (26) reproduces those results. We emphasize that the flow equations obtained for the alternative "side" geometry ${ }^{51,52}$ differ from the present "tunneling" geometry ones by a relevant scale dimension $1>K$ of the inter-lead couplings rather than the irrelevant scale dimension $1 / K>1$ (as $r>0$ ) found above. The resulting physics is thus markedly different for the two cases.

\section{Strong-coupling analysis}

The low-energy physics and therefore transport and thermodynamic properties are dominated by the vicinity of the strong-coupling fixed point. In order to analyze the low-energy physics, two distinctive regimes arise depending on the value of $r$.

Small values $r<1 / 2$. The previous weak-coupling analysis has shown that all Kondo interaction terms are driven to strong coupling such that the usual one-channel Kondo physics should a priori be recovered. However, for a more precise statement the vicinity of this fixed point should be considered. A detailed analysis shows that particle-hole symmetry-breaking terms are relevant at the one-channel Kondo fixed point. One indeed finds that potential scattering terms like $V c^{\dagger} c$ in Eq. (19) have a scaling dimension $(1+K) / 2=(1+r) /(1+2 r)<1$ and are therefore relevant. In a one-dimensional infinite Luttinger liquid chain, such a term "cuts" the chain into two semi-infinite chains below an energy scale $T^{*} \cdot 37$ Therefore, the ultimate fixed point which the system reaches for $T=e V=0$ corresponds to an insulating one $\frac{38,39,40}{{ }^{3}}$ The analysis of the operator content at this insulating fixed point allows to predict the scaling behavior with temperature or bias voltage. Here we focus on the differential conductance $d I / d V$ between the left and right lead. The leading irrelevant operator corresponds to a hopping term between the two leads and has dimension $(1+1 / K) / 2=1+r>1$. We note that particle-hole symmetry-breaking effects can be tuned to zero, using the dot gate voltage. The Kondo resonance may therefore develop in transport for some particular value of the gate voltage $V_{g}^{*}$, as specified in Eq. (29b) below. The finitetemperature corrections to the unitary limit are given by the leading irrelevant operator nearby this perfectly transmitting fixed point when particle-hole symmetry is imposed. This operator has dimension $2 K$ and is irrelevant for $K>1 / 2{ }^{39}$ These results imply an anomalous behavior of the differential conductance at low temperature:

$$
\begin{aligned}
& \frac{d I}{d V} \approx a\left(\frac{e V}{T^{*}}\right)^{2 r} \text { for } V_{g} \neq V_{g}^{*} \\
& \frac{d I}{d V} \approx 2 G_{K}\left[1-b\left(\frac{e V}{T_{K}}\right)^{2 r}\right] \text { for } V_{g}=V_{g}^{*}
\end{aligned}
$$

with $G_{K}=1 / R_{K}=e^{2} / h$, and $a, b$ dimensionless nonuniversal prefactors. At finite temperature $T$ and zero bias voltage, the energy $\mathrm{eV}$ should be replaced by $T$ in Eqs. (29). The conductance in Eq. (29a) is similar to the conductance through a single tunnel junction. One may also wonder how the conductance is modified when $V_{g}$ slightly deviates from $V_{g}^{*}$. Since particle-hole symmetry is relevant at this perfectly transmitting fixed point, and the resonance is very sharp. Following Ref. [37], it can be shown that the width of the resonance scales as $T^{(1-K) / 2}$ where $V_{g}$ deviates from $V_{g}^{*}$. The differential conductance escapes from the unitary limit as follows

$$
\frac{d I}{d V} \approx 2 G_{K}\left[1-b\left(\frac{\Gamma}{e V}\right)^{\frac{2 r}{1+2 r}}\right] \text { for } V_{g} \approx V_{g}^{*}
$$

where $\Gamma$ is a characteristic energy scale related to $\delta V_{g}=$ $V_{g}-V_{g}^{*}$, which vanishes as $\delta V_{g}^{2 /(1-K)}$ at resonance $\frac{37}{4}$

Large values $r>1 / 2$. For large values of $r$, dissipation dominates the renormalization-group flow in Eq. (27a) and the Kondo coupling $J_{L R}$ renormalizes to zero. Therefore coherent charge transfer between left and right lead is suppressed, without however completely inhibiting the Kondo effect, similarly to what occurs in the case of strongly Coulomb blockaded leads ${ }^{42,43}$. Indeed the Kondo couplings $J_{L L}$ and $J_{R R}$ are still driven to strong coupling according to Eq. (27b). A two-channel Kondo fixed point can be reached provided $J_{L L}=J_{R R}$. By analyzing the operator content of the strong-coupling two-channel fixed point, it has been shown to be stable against particle-hole symmetry-breaking terms for 
$K<1 / 2$, or equivalently $r>1 / 2 \cdot 38,39$ Indeed, the leading irrelevant operator near the two-channel Kondo fixed point occurs in the flavor sector and has dimension $1 /(2 K)=2 r+1$, and is therefore irrelevant for $r>1 / 2 \underline{39}$ This operator will therefore dominate the transport properties below the two-channel Kondo temperature $T_{2 C K}$. For $J_{L L} \neq J_{R R}$ the two-channel Kondo fixed point is unstable and a single-channel Kondo effect occurs in the strongest coupled channel (either left or right). The transport for this situation is characterized by the leading irrelevant operator corresponding to a hopping term between the two leads as for $r<1 / 2$ with dimension $(1+1 / K) / 2=1+r>1$. From this above analysis we can infer the anomalous behavior of the differential conductance $d I / d V$ between the left and right leads at low energy:

$$
\begin{aligned}
& \frac{d I}{d V} \approx c\left(\frac{e V}{T_{1 C K}}\right)^{2 r} \text { for } J_{L L} \neq J_{R R}, \\
& \frac{d I}{d V} \approx d\left(\frac{e V}{T_{2 C K}}\right)^{2 r-1} \text { for } J_{L L}=J_{R R},
\end{aligned}
$$

where $c, d$ are nonuniversal numbers and $T_{1 C K}$ is the onechannel (eithr left or right) Kondo temperature. Notice the absence of $G_{K}$, peculiar to the two-channel Kondo effect in Eq. (31b), due to the tunneling geometry. A third, nonnoisy electrode would be necessary to obtain this behavior, see Ref. [42,43].

This analysis can be directly extended to a quantum dot operating as a spin- 1 impurity. The low-energy physics resembles then that of a spin- 1 impurity embedded between two Luttinger liquids, recently studied in Ref. [53].

\section{CONCLUSION}

In this paper we investigated the impact of environmental Coulomb blockade on the Kondo effect in the case of moderate to highly resistive leads. An ohmic resistance of the environment exceeding $R_{K} / 2$ induces a suppression of the inter-lead Kondo interactions, without however preventing the formation of a strong-coupling state, due to the remaining intra-lead processes. When the tunneling amplitudes between the left and right leads are equal, even a two-channel Kondo effect can be reached. For an environmental resistance smaller than $R_{K} / 2$ the Kondo effect can fully develop between both leads. However, the fully transparent fixed point is stable only when particle-hole symmetry is maintained with the dot plunger gate voltage. On a qualitative level these results imply anomalous low-temperature transport properties through the device, even though the Kondo effect survives. More quantitatively, the Kondo temperature below which those features start to appear was shown to depend sensitively not only on the dissipation strength, but rather on the full spectral function of the excited environmental modes. Although strong-resistive effects may be difficult to detect in actual experiments, due to the difficulty in the realization of well-coupled highly dissipative setups in semiconducting quantum dots, such a reduction of the Kondo temperature at moderate dissipation as well as nonlinearities in the $\mathrm{I}(\mathrm{V})$ characteristics should be observed.

Theoretically, it would be interesting to go beyond the quasi-elastic approximation by which our phenomenological Kondo model is applicable. This approximation may rather underestimate the environmental effects. A generalization of this study to orbital Kondo effects or mixed spin-orbital Kondo setups can also be performed along the same line $\mathrm{e}^{54}$ merging the present situation with the previously studied decoherence effects on the Kondo effect in the charge sector.

\section{Acknowledgments}

This work has been supported by the French ANR (program PNano), the DFG Center for Functional Nanostructures in Karlsruhe, and the Virtual Quantum Phase Transitions Institute in Karlsruhe. We would also like to acknowledge valuable discussions with K. Le Hur at the early stages of this work.

\section{APPENDIX A: EQUIVALENCE BETWEEN THE NOISY KONDO PROBLEM AND THE KONDO MODEL IN LUTTINGER LIQUIDS}

We provide here the derivation for the low-energy mapping of the Kondo model (17) in an ohmic environment onto the usual Kondo model between Luttinger liquids.

The free electronic part of Eq. (14) is first conveniently expressed as a chiral Hamiltonian for left and right electrodes

$$
H_{\text {leads }}=-i v_{F} \int_{-\infty}^{+\infty} d x \sum_{\sigma \alpha} c_{\sigma, \alpha}^{\dagger} \partial_{x} c_{\sigma, \alpha}
$$

We bosonize each channel, keeping the Klein factors explicitely

$$
c_{\sigma \alpha}=\frac{1}{\sqrt{2 \pi a}} F_{\sigma \alpha} \exp \left(i \frac{\phi_{c \alpha}+(-1)^{\sigma} \phi_{s \alpha}}{\sqrt{2}}\right) .
$$

This allows to express the above Eq. (A1) in terms of charge and spin bosons in each channel

$$
H_{\text {leads }}=\frac{v_{F}}{4 \pi} \int_{-\infty}^{+\infty} d x \sum_{\alpha}\left(\partial_{x} \phi_{c \alpha}\right)^{2}+\left(\partial_{x} \phi_{s \alpha}\right)^{2} .
$$

Using the identity for the electron density operator

$$
c_{\sigma \alpha}^{\dagger} c_{\sigma \alpha}=\frac{1}{2 \pi} \frac{\partial_{x} \phi_{c \alpha}+(-1)^{\sigma} \partial_{x} \phi_{s \alpha}}{\sqrt{2}}
$$


and the bosonization dictionary (A2), we can now reexpress the Kondo Hamiltonian (17). To clarify the resulting expression, we define the following basis change between the $L, R$ channels

$$
\begin{aligned}
\phi_{c} & \equiv \frac{\phi_{c L}+\phi_{c R}}{\sqrt{2}} \\
\phi_{f} & \equiv \frac{\phi_{c L}-\phi_{c R}}{\sqrt{2}} \\
\phi_{s} & \equiv \frac{\phi_{s L}+\phi_{s R}}{\sqrt{2}} \\
\phi_{s f} & \equiv \frac{\phi_{s L}-\phi_{s R}}{\sqrt{2}} .
\end{aligned}
$$

We have also to perform a similar transformation on the Klein factors 55 and introduce for this purpose four alternate Klein fermions, $F_{c}, F_{f}, F_{s}, F_{s f}$, which obey

$$
\begin{aligned}
F_{s f}^{\dagger} F_{s}^{\dagger} & =F_{e \uparrow}^{\dagger} F_{e \downarrow} \\
F_{s f} F_{s}^{\dagger} & =F_{2 \uparrow}^{\dagger} F_{2 \downarrow} \\
F_{s f}^{\dagger} F_{f}^{\dagger} & =F_{e \uparrow}^{\dagger} F_{2 \uparrow} \\
F_{c}^{\dagger} F_{s} & =F_{e \uparrow}^{\dagger} F_{2 \uparrow}^{\dagger} .
\end{aligned}
$$

All other bilinears of the original Klein factors are easily computed from the above expression, e.g. $F_{e \downarrow}^{\dagger} F_{2 \uparrow}=$ $F_{e \downarrow}^{\dagger} F_{e \uparrow} F_{e \uparrow}^{\dagger} F_{2 \uparrow}=F_{s} F_{s f} F_{s f}^{\dagger} F_{f}^{\dagger}=F_{s} F_{f}^{\dagger}$.

We introduce also spin anisotropies of the exchange constants, with the new notation $\left(J_{F}^{\perp}, J_{F}^{z}, J_{B}^{\perp}, J_{B}^{z}\right)$, where the subscript $F(B)$ stands for $J_{L L}=J_{R R}\left(J_{L R}=\right.$ $\left.J_{R L}\right)$, and the superscript $\perp(z)$ for the $x, y(z)$ spin orientations respectively. We assumed here perfect symmetry between the left and right couplings not to overburden the notation, although this will not affect the validity of the mapping we want to establish. The final expression for the Kondo term (17) then reads

$$
\begin{aligned}
H_{K} & =\frac{J_{F}^{\perp}}{4 \pi a} S^{+} F_{s} e^{i \phi_{s}}\left(F_{s f} e^{i \phi_{s f}}+F_{s f}^{\dagger} e^{-i \phi_{s f}}\right)+\text { H.c. } \\
& +\frac{J_{F}^{z}}{2 \pi} S^{z} \partial_{x} \phi_{s} \\
& +\frac{J_{B}^{\perp}}{4 \pi a} S^{+} F_{s} e^{i \phi_{s}}\left(F_{f} e^{i \phi_{f}+i \varphi}+F_{f}^{\dagger} e^{-i \phi_{f}-i \varphi}\right)+\text { H.c. } \\
& +\frac{J_{B}^{z}}{4 \pi a} S^{z} F_{f}^{\dagger} e^{-i \phi_{f}-i \varphi}\left(F_{s f}^{\dagger} e^{-i \phi_{s f}}-F_{s f} e^{i \phi_{s f}}\right)+\text { H.c. }
\end{aligned}
$$

We see that the environmental boson $\varphi$ occurs naturally as a systematic shift of the "flavor" field $\phi_{f}$ describing the charge transfer between the two electrodes. To define a simple effective environment, we consider the combined effective action for both the local density bosons (integrating all $x \neq 0$ in Eq. (A3)), and the environmental boson from Eq. (2)

$$
\begin{aligned}
S_{0} & =\frac{1}{\beta} \sum_{\nu_{n}}\left|\nu_{n}\right|\left(\left|\phi_{c}\right|^{2}+\left|\phi_{f}\right|^{2}+\left|\phi_{s}\right|^{2}+\left|\phi_{s f}\right|^{2}+\frac{R_{K}}{2 R}|\varphi|^{2}\right) \\
& =\frac{1}{\beta} \sum_{\nu_{n}}\left|\nu_{n}\right|\left(\left|\phi_{c}\right|^{2}+\left|\phi_{f}^{\prime}\right|^{2}+\left|\phi_{s}\right|^{2}+\left|\phi_{s f}\right|^{2}+\left|\varphi^{\prime}\right|^{2}\right),
\end{aligned}
$$

where

$$
\begin{aligned}
\phi_{f}^{\prime} & =\sqrt{K}\left(\phi_{f}+\varphi\right) \\
\varphi^{\prime} & =\sqrt{K}\left(\sqrt{\frac{2 R}{R_{K}}} \phi_{f}-\sqrt{\frac{R_{K}}{2 R}} \varphi\right)
\end{aligned}
$$

and

$$
K \equiv \frac{1}{1+2 R / R_{K}}
$$

Therefore the role of the ohmic environment on the Kondo effect is to replace all exponential terms $e^{i \phi_{f}}$ in the bosonized expression by $e^{i \phi_{f}^{\prime} / \sqrt{K}}$. Starting from a standard magnetic impurity between two Luttinger liquid leads characterized by an interaction parameter $K$ (without environmental modes), with the open boundary bosonization condition, 38 leads to the same final expression, thus proving the desired equivalence.
1 D. Goldhaber-Gordon, H. Shtrikman, D. Mahalu, D. Abusch-Magder, U. Meirav, and M. A. Kastner, Nature 391, 156 (1998).

2 L. Kouwenhoven and L. Glazman, Physics World 14, 33 (2001).

3 L. I. Glazman and M. E. Raikh, JETP Lett. 47, 452 (1988); T. K. Ng, P. A. Lee, Phys. Rev. Lett. 61, 1768 (1988).
4 A. Kogan, S. Amasha, and M. A. Kastner, Science 304, 1293 (2004).

5 N. S. Wingreen and Y. Meir, Phys. Rev. B 49, 11040 (1994).

6 A. Kaminski, Y. V. Nazarov, and L. I. Glazman, Phys. Rev. B 62, 8154 (2000).

7 P. Simon and I. Affleck, Phys. Rev. Lett. 89, 206602 
(2002).

8 R. K. Kaul, G. Zaránd, S. Chandrasekharan, D. Ullmo, and H. U. Baranger, Phys. Rev. Lett. 96, 176802 (2006); P. Simon, J. Salomez, and D. Feinberg, Phys. Rev. B 73, 205325 (2006).

9 M. H. Devoret and H. Grabert, in "Single Charge Tunneling", edited by H. Grabert and M. H. Devoret, NATO ASI Series B, Vol. 294, pp. 1-19 (Plenum Press, New York, 1992).

10 G.-L. Ingold and Yu. V. Nazarov, in "Single Charge Tunneling", edited by H. Grabert and M. H. Devoret, NATO ASI Series B, Vol. 294, pp. 21-107 (Plenum Press, New York, 1992).

11 P. Delsing, K. K. Likarev, L. S. Kuzmin, and T. Claeson, Phys. Rev. Lett. 63, 1180 (1989).

12 A. N. Cleland, J. M. Schmidt, and J. Clarke, Phys. Rev. Lett. 64, 1565 (1990).

13 P. Joyez, D. Esteve, and M. H. Devoret, Phys. Rev. Lett. 80, 1956 (1998).

14 Yu. V. Nazarov, Zh. Eksp. Teor. Fiz. 95, 975 (1989) [Sov. Phys. JETP 68, 561 (1989)].

15 S. M. Girvin, L. I. Glazman, M. Jonson, D. R. Penn, and M. D. Stiles, Phys. Rev. Lett. 64, 3183 (1990).

16 A. A. Odintsov, G. Falci, and G. Schön, Phys. Rev. B 44, 13089 (1991).

17 M. H. Devoret, D. Esteve, H. Grabert, G.-L. Ingold, H. Pothier, and C. Urbina, Phys. Rev. Lett. 64, 1824 (1990).

18 K. Flensberg and M. Jonson, Phys. Rev. B, 43, 7586 (1991).

19 S.V. Panyukov and A.D. Zaikin, Phys. Rev. Lett. bf 67, 3168 (1991).

20 X. Wang and H. Grabert, Phys. Rev. B 53, 12621 (1996).

21 I. Safi and H. Saleur, Phys. Rev. Lett. 93, 126602 (2004).

22 A. L. Yeyati, A. Martin-Rodero, D. Esteve, and C. Urbina, Phys. Rev. Lett. 87, 046802 (2001).

${ }^{23}$ Yu. V. Nazarov, Phys. Rev. Lett. 82, 1245 (1999).

24 D. S. Golubev and A. D. Zaikin, Phys. Rev. Lett. 86, 4887 (2001).

25 A. A. Odintsov, V. Bubanja, and G. Schön, Phys. Rev. B 46, 6875 (1992).

${ }^{26}$ P. Joyez and D. Esteve, Phys. Rev. B 56, 1848 (1997).

27 G. Schön and A.D. Zaikin, Phys. Rep. 198, 237 (1990).

28 M. Watanabe and D. B. Haviland, Phys. Rev. Lett. 86, 5120 (2001).

29 A. J. Rimberg, T. R. Ho, Ç. Kurdak, J. Clarke, K. L. Campman, and A. C. Gossard, Phys. Rev. Lett. 78, 2632 (1997).

30 D. Popovic, C. J. B. Ford, J. M. Hong, and A. B. Fowler, Phys. Rev. B 48, 12349 (1993).

31 K. Flensberg, Phys. Rev. B 48, 11156 (1993).
32 K. A. Matveev, Phys. Rev. B 51, 1743 (1995).

${ }^{33}$ K. Le Hur, Phys. Rev. Lett. 92, 196804 (2004).

${ }^{34}$ K. Le Hur and M.-R. Li, Phys. Rev. B 72, 073305 (2005).

35 L. Borda, G. Zarand, and P. Simon, Phys. Rev. B 72, 155311 (2005).

36 L. Borda, G. Zarand, and D. Goldhaber-Gordon, preprint cond-mat/0602019 (unpublished).

37 C. L. Kane and M. P. A. Fisher, Phys. Rev. B 46, 15233 (1992).

38 M. Fabrizio and A. O. Gogolin, Phys. Rev. B 5117827 (1995).

39 E. H. Kim, cond-mat/0106575 (unpublished).

40 P. Simon and I. Affleck, Phys. Rev. B 64, 085308 (2001).

41 S. Andergassen, T. Enss, and V. Meden, Phys. Rev. B 73, 153308 (2006).

42 Y. Oreg and D. Goldhaber-Gordon, Phys. Rev. Lett. 90, 136602 (2003).

43 S. Florens and A. Rosch, Phys. Rev. Lett. 92, 216601 (2004).

44 R. M. Potok, I. G. Rau, H. Shtrikman, Y. Oreg, D. Goldhaber-Gordon, preprint cond-mat/0610721 (unpublished).

45 A. O. Caldeira and A. J. Leggett, Annals of Physics 149, 374 (1983); A. J. Leggett et al., Rev. Mod. Phys. 59, 1 (1987).

46 R. Cron, PhD Thesis, 2001, University of Paris VI, Paris, France. http://www-drecam.cea.fr/articles/s01/099/publi.pdf

47 H. T. Imam, V. V. Ponomarenko, and D. V. Averin, Phys. Rev. B 50, 18288 (1994).

48 G.L. Ingold, P. Wyrovski, and H. Grabert, Z. Phys. B 85, 443 (1991).

49 D. V. Averin and Yu. Z. Nazarov, Physica B 189, 214 (1993).

50 J. Paaske and K. Flensberg, Phys. Rev. Lett. 94, 176801 (2005).

51 D.-H. Lee and J. Toner, Phys. Rev. Lett. 69, 3378 (1992).

52 A. Furusaki and N. Nagaosa, Phys. Rev. Lett. 72, 892 (1994).

53 P. Durganandini and P. Simon, Phys. Rev. B 74, 205304 (2006).

54 S. Andergassen, S. Florens, P. Simon and D. Feinberg (in preparation).

55 G. Zaránd and J. von Delft, Phys. Rev. B 61, 006918 (2000).

56 The matching of low and high energy behavior of $P(E)$, Eqs. (10) and (11) respectively, gives indeed a regime change at $\omega_{c}$ for $r \ll 1$, while this occurs at the scale $\pi r \omega_{c}=E_{c}$ for $r \gg 1$. 